hep-th/9607057

PUPT-1636

TIFR/TH/96-37

July 1996

\title{
Orbifold and Orientifold Compactifications of F-Theory and M-Theory to Six and Four Dimensions
}

\author{
Rajesh Gopakumari \\ Department of Physics, Princeton University, \\ Princeton, NJ 08544, U.S.A. \\ and \\ Sunil Mukhi目 \\ Tata Institute of Fundamental Research, \\ Homi Bhabha Rd, Mumbai 400 005, India
}

\begin{abstract}
We study orbifold compactifications of F-theory which lead to $N=1$ supersymmetry in 6 and 4 spacetime dimensions. These are dual to specific orientifolds of M-theory, and in many cases to orientifolds of type IIB string theory. The equivalences are demonstrated by mapping the orbifolding transformations in the F, M and string theories to each other using dualities. We observe that $\mathrm{M}$ and $\mathrm{F}$-theory appear to possess a property similar to discrete torsion in string theory. This is related to an ambiguity recently noted by Polchinski in the orientifold projection for 6-dimensional models. The 4-dimensional compactifications exhibit similar features, from which we predict the existence of certain new orientifolds of type IIB. Some orbifolds with higher supersymmetry are also examined.
\end{abstract}

\footnotetext{
1 E-mail: gopakumr@phoenix.princeton.edu

2 E-mail: mukhi@theory.tifr.res.in
} 


\section{Introduction}

Many physical and mathematical phenomena that have been regarded as unique to string theory turn out to have deeper explanations in terms of M-theory [1] and F-theory [2].

Compactifications of these new theories are in general difficult to describe, since we presently lack a formulation of these theories with the same power as perturbative string theory. However, in string theory it has often been the case that such compactifications are more easily studied for orbifolds[3] (and their generalizations, orientifolds [4] [5]). Recently it has become evident that compactifications of $\mathrm{M}$ and F-theory on these spaces can also be discussed in a surprising amount of detail.

The first example of this sort was the orientifold of M-theory on $S^{1} / Z_{2}$, shown by Horava and Witten [6] to be dual to the $E_{8} \times E_{8}$ heterotic string in 10 dimensions. This example turns out not to be sufficiently generic; for example, this is a case where orbifolding does not commute with duality[7]. The first generic examples were the orientifolds of M-theory on $T^{5} / Z_{2}$ down to 6 dimensions, and on $T^{9} / Z_{2}$ down to 2 dimensions, described in Ref.[8]. These were argued to be dual to the type IIB string compactified on K3 and $T^{8} / Z_{2}$ respectively. The $T^{5} / Z_{2}$ case, independently studied in Ref. [9], has another interesting property: the vacuum contains 5-branes of M-theory, which carry the requisite matter multiplets to cancel the anomaly in 6d. Subsequently, a number of M-theory orientifolds [10] 11] [7] [12 and orbifolds 13] [14] have been investigated. (We distinguish between orbifold and orientifold compactifications of M-theory by the fact that the latter have an orientiation-reversing action on the compactification torus and reverse the M-theory 3-form.)

For F-theory, orbifold limits of certain Calabi-Yau 3-fold compactifications were discussed in Ref. 15. Orbifold limits of the original K3 compactification have been recently investigated in some detail in Refs. [16] [17]. Most of the examples discussed in the above references dealt with compactification down to 8 or 6 spacetime dimensions.

Studying orbifold/orientifold compactifications of $\mathrm{F}$ and $\mathrm{M}$ theory is likely to be particularly useful because we still lack a satisfactory fundamental formulation of these theories. Such a formulation would also presumably be a fundamental non-perturbative formulation of string theory. We may hope to gain some insight into this problem by using flat but singular compactifying spaces.

A distinct but closely related programme pursued in recent times has been the study of orientifolds of string theory [18] [19] [20] [21] [22] [23] [24]. These have the advantage of being 
free conformal field theory realisations of non-perturbative vacua. Some inter-relationships among orientifolds of string theory, and orbifolds/orientifolds of $\mathrm{M}$ and $\mathrm{F}$ theories, were investigated in Refs. [7] [16][17.

In what follows, we study a class of F-theory compactifications on Calabi-Yau threefolds and fourfolds down to 6 and 4 spacetime dimensions. These give rise in each case to vacua with the minimum supersymmetry compatible with the number of spacetime dimensions. The special property of the manifolds we study is the fact that they admit orbifold limits in their moduli space. It is then possible to establish an equivalence to orientifolds of M-theory of the generic form $T^{5} /\left(Z_{2}\right)^{2}$ and $T^{7} /\left(Z_{2}\right)^{3}$ respectively. These dualities can also be extended to produce type IIB orientifold duals, some of which have been independently investigated in the various recent works cited above. We will see that while the map between F-theory and IIB orientifolds is perturbative, that to M-theory is not. This allows us to view these vacua often from complementary viewpoints. This is potentially useful in understanding interesting objects like tensionless strings which play a role in phase transitions. One example of this utility will be in examining an ambiguity in IIB orientifolds that we will describe later. Our mapping also leads us to believe that many M-theory orientifold vacua may be realised in F-theory.

In the four-dimensional case, we actually study a much larger class of non-toroidal M-theory orientifolds, of the form $C Y_{3} \times S^{1} / Z_{2},\left(K 3 \times T^{2} \times S^{1} / Z_{2}\right) /\left(Z_{2}\right)$ etc., for which again the equivalences to $F$ theory and IIB orientifolds are demonstrated. These compactifications all have $\mathrm{N}=1$ spacetime supersymmetry in $4 \mathrm{~d}$. Given the potential importance of four-dimensional $\mathrm{N}=1$ supersymmetry to present-day particle physics, it is clearly desirable to examine properties of the most solvable cases in some detail. We believe the cases at hand fall into this category.

We find that for orbifolding groups larger than $Z_{2}$, the projection of twisted-sector states of one element onto the subspace invariant under the other elements sometimes presents ambiguities. In particular for the group $Z_{2} \times Z_{2}$ we find a model with a twofold ambiguity, leading to two distinct vacua. The phenomenon has a close operational resemblance to discrete torsion in string theory, and we develop the analogy in some detail. For $Z_{2} \times Z_{2} \times Z_{2}$ models there is apparently a fourfold ambiguity. We relate the twofold ambiguity to a phenomenon discussed recently by Polchinski for string theory on orientifolds to six dimensions. The fourfold ambiguity appears to be a combination of the above phenomenon and conventional discrete torsion, and seems to indicate that the two must enter on an equal footing in F-theory or M-theory. 
Most, but not all, of the compactifications of F-theory to 4 dimensions that we define pass the consistency test of Ref. [25], according to which the Euler characteristic $\chi$ should be a positive integral multiple of 24 , so that tadpoles can be cancelled by 3 -branes in the vacuum. We will find one model with $\chi=0$, for which no such 3 -branes are required.

This paper is organized as follows. In Sections 2 and 3 we define and analyse orbifold/orientifold compactifications of $\mathrm{F}$ and $\mathrm{M}$ theory to 6 dimensions, and demonstrate their equivalence to each other and to orientifolds of type IIB. The ambiguity referred to above is discussed in Section 3. In Sections 4 and 5 we discuss analogous compactifications to 4 dimensions. We make a number of predictions which could be verified by further investigations of type IIB orientifolds. In section 6 we discuss some orientifolds and orbifolds of $\mathrm{M}$ and $\mathrm{F}$ theory which lead to $N>1$ supersymmetry in 4 dimensions. Finally, in Section 7 we present the conclusions and describe some open questions for the future.

\section{Compactifications to six dimensions}

We start by describing a set of three Calabi-Yau complex threefolds, all of which contain a point in their moduli space where they can be realised as orbifolds of the form $T^{6} /\left(Z_{2} \times Z_{2}\right)$. All of them are actually members of the Voisin-Borcea family of CalabiYau 3-folds [26] [27, and have already been studied in connection with string theory and F-theory compactifications in Ref.[15].

In what follows we shall study F-theory on these manifolds. This may be regarded as IIB compactified on the base $T^{4} /\left(Z_{2} \times Z_{2}\right)$ with the $T^{2}$ fibre typically degenerating to $T^{2} / Z_{2}$ on fixed points/curves on this base. Let us briefly recapitulate how to obtain the spectrum for F-theory on Calabi-Yau 3-folds[15]. Upon further compactification on $T^{2}$, the theory is on the same moduli space as IIA on the 3-fold. This immediately tells us that the number of neutral hypermultiplets is

$$
h^{0}=H^{2,1}(X)+1 .
$$

The tensor multiplets are determined by the Kahler deformations that preserve the elliptic fibration. Moreover since vectors and tensors of $\mathrm{N}=1$ in $6 \mathrm{~d}$ on compactification give vectors of $\mathrm{N}=2$ in $4 \mathrm{~d}$, we have

$$
T=H^{1,1}(B)-1, \quad r(V)=H^{1,1}(X)-H^{1,1}(B)-1
$$


Here $X$ and $B$ refer to the 3 -fold and the base of the fibration respectively, $T$ is the number of $\mathrm{N}=1$ tensor multiplets and $r(V)$ is the rank of the non-abelian gauge group. The gauge group itself is determined by the singularity type of the degenerating fibre. There are subtleties in this dictionary [28], but we will choose to work in the region of moduli space where the naive gauge group is realised.

In what follows, we label the spacetime coordinates $x^{1}, x^{2}, \ldots$ where $x^{1}$ is the time. The orbifolds can be defined as follows: each of the three non-trivial elements of order 2 in the orbifolding group $Z_{2} \times Z_{2}$ reverses the sign of 4 of the 6 torus coordinates. Labelling these three elements $\alpha, \beta$ and $\alpha \beta$, the orbifold action is given by specifying which of the 6 coordinates $x^{7}, x^{8}, \ldots, x^{12}$ is inverted, and whether the coordinate also gets a shift by a $1 / 2$-unit. The "coordinates" $x^{11}, x^{12}$ will be taken to be those of the fibre $T^{2}$. There are three inequivalent choices which give us in turn the following models, labelled by their Hodge numbers $\left(H^{1,1}, H^{2,1}\right)$ :

Model A: $(11,11)$

\begin{tabular}{|c|c|c|c|c|c|c|}
\hline & $x^{12}$ & $x^{11}$ & $x^{10}$ & $x^{9}$ & $x^{8}$ & $x^{7}$ \\
\hline$\alpha$ & + & + & - & - & - & - \\
\hline$\beta$ & - & - &,$+ \frac{1}{2}$ & + &,$- \frac{1}{2}$ & - \\
\hline$\alpha \beta$ & - & - &,$- \frac{1}{2}$ & - &,$+ \frac{1}{2}$ & + \\
\hline
\end{tabular}

This manifold has also featured in Type II compactifications [29]. The base is the so-called Enriques surface. Note that it has no fixed points where the fibre degenerates. Hence one expects no gauge fields at all. The spectrum thus consists of 9 tensor and 12 hypermultiplets. The same spectrum also appears in type IIB on a $T^{4} / Z_{4}$ orientifold[20][21].

Model B: $(19,19)$

\begin{tabular}{|c|c|c|c|c|c|c|}
\hline & $x^{12}$ & $x^{11}$ & $x^{10}$ & $x^{9}$ & $x^{8}$ & $x^{7}$ \\
\hline$\alpha$ & + & + & - & - & - & - \\
\hline$\beta$ & - & - &,$+ \frac{1}{2}$ & + & - & - \\
\hline$\alpha \beta$ & - & - &,$- \frac{1}{2}$ & - & + & + \\
\hline
\end{tabular}

Here we see that the fibre degenerates to $T^{2} / Z_{2}$ at the four fixed points of $\alpha \beta$. The monodromy around these points is precisely of the form studied in 16 . We know that it is a $D_{4}$ singularity and results in an $S O(8)$ enhanced symmetry coming from four coincident 7 -branes located at each fixed point (and wrapped around $x^{7}, x^{8}$ ). The four fixed points 
are, however, permuted pairwise by the action of $\beta$, and thus we only get an $S O(8)^{2}$ enhanced symmetry. By moving the 7-branes off the fixed points, we generically break the symmetry to $U(1)^{8}$. Thus at generic points in its moduli space, this model has 9 tensor and 20 neutral hypermultiplets. The Higgsing at the orbifold point was accomplished by 2 hypermultiplets in the adjoint of $S O(8)$. The gauge symmetries can be enhanced at other special points of moduli space as well, with the addition of charged hypermultiplets. This spectrum coincides with that of an M-theory orbifold [10] and a IIB orientifold [19]. Later we will demonstrate the equivalence of all three realisations.

Model C: $(51,3)$

\begin{tabular}{|c|c|c|c|c|c|c|}
\hline & $x^{12}$ & $x^{11}$ & $x^{10}$ & $x^{9}$ & $x^{8}$ & $x^{7}$ \\
\hline$\alpha$ & + & + & - & - & - & - \\
\hline$\beta$ & - & - & + & + & - & - \\
\hline$\alpha \beta$ & - & - & - & - & + & + \\
\hline
\end{tabular}

This last model shows many intriguing features, as we will see. First note that the the base $T^{4} /\left(Z_{2} \times Z_{2}\right)$ is actually $T^{2} / Z_{2} \times T^{2} / Z_{2}$ with the $Z_{2}$ 's generated by $\beta$ and $\gamma$. The fibre degenerates over $4+4$ curves of the base in the manner of [16]. There are also $4 \times 4$ fixed points on the base, which can be blown up and thus contribute 16 to the total $H^{1,1}(B)=18$. Hence we have an anomaly-free spectrum consisting of $S O(8)^{8}$ vector multiplets, 17 tensor multiplets and 4 neutral hypermultiplets. The absence of charged hypermultiplets implies that the gauge group is generically non-abelian [15] and can even be further enhanced.

The fact that the base looks like a singular limit of $P^{1} \times P^{1}$ suggests a connection with another Calabi-Yau, the $(3,243)$, which admits an elliptic fibration over $P^{1} \times P^{1}$. This latter model is dual to the $E_{8} \times E_{8}$ heterotic string on $K 3$ with symmetric instanton embedding[30] [31]. This connection will be examined in more detail below.

As we noted above, for the (19,19), there is a five-dimensional M-theory orientifold[10], and also a type IIB orientifold[19] with the same spectrum. M-theory duals to the other two cases have not so far been identified. We now show that there are precisely three M-theory orientifolds of the form $T^{5} /\left(Z_{2} \times Z_{2}\right)$ which give $\mathrm{N}=1$ supersymmetry in $6 \mathrm{~d}$, and can be put in a natural correspondence with the Calabi-Yau orbifolds A, B and C of F-theory described above, and that M-theory compactified on each of them can be mapped in a precise way to F-theory on the corresponding Calabi-Yau. 
To this end, first note that in the $(11,11)$, only one of the three nontrivial elements of the orbifold group has fixed loci, while in the $(19,19)$, two elements have fixed loci and in the (51,3), all three have fixed loci. For an M-theory orbifold to have $\mathrm{N}=1$ supersymmetry one also needs two $Z_{2}$ actions, each of which reduces the supersymmetry by half. It is easy to see that this can only happen if the number of coordinate inversions is 0 or 1 mod 4 . This forces the three nontrivial elements of $Z_{2} \times Z_{2}$ to consist of 5,4 and 1 inversion in turn. It remains to incorporate shifts in appropriate ways so that one is led to three models where one, two and three elements act with fixed points. Thus we are led to consider M-theory on the three orbifolds (more properly labelled as orientifolds), of the form $T^{5} / Z_{2} \times Z_{2}$ defined below. The spectra of these three models will also be worked out in the following. The untwisted sector is common to all three models and is easily shown to consist of one tensor and four neutral hypermultiplets. Thus we need only examine the twisted sector separately for each case.

Model $\mathrm{A}^{\prime}: T^{5} /\left(Z_{2} \times Z_{2}\right)$

\begin{tabular}{|c|c|c|c|c|c|}
\hline & $x^{11}$ & $x^{10}$ & $x^{9}$ & $x^{8}$ & $x^{7}$ \\
\hline$\alpha$ & - & - & - & - & - \\
\hline$\beta$ &,$+ \frac{1}{2}$ & - & - &,$- \frac{1}{2}$ & - \\
\hline$\alpha \beta$ &,$- \frac{1}{2}$ & + & + &,$+ \frac{1}{2}$ & + \\
\hline
\end{tabular}

The element $\alpha$ is the only element that acts with fixed points. It's action on $T^{5}$ leads to the $T^{5} / Z_{2}$ orbifold of M-theory [8] [9]. The element $\beta$ is seen to be a shift in the 11th direction and an action on the remaining $T^{4}$, which is identical to the involution on $T^{4} / Z_{2}$ considered in 32 33. The twisted sector of $\alpha$ has been argued to consist of 16 M-theory 5-branes [9], each carrying a multiplet that in $N=1$ supersymmetry is the sum of a tensor and a hypermultiplet. In the present case, this twisted-sector multiplet needs to be projected onto the sector invariant under $\beta$ and $\gamma$. Since the twisted-sector states arise from 5-branes, it is natural to suppose that the branes are to be located at 16 of the fixed points of the orientifold, and the action of $\beta$ and $\gamma$ on them is just the respective geometrical action. Because of the shifts, this means that the 165 -branes get permuted amongst each other, with the result that half of them are odd and get projected out. This leaves 8 tensor and 8 hypermultiplets, which when combined with the untwisted sector give precisely the same spectrum as for F-theory on model A. 
Model $\mathrm{B}^{\prime}:\left(K 3 \times S^{1}\right) / Z_{2}$

\begin{tabular}{|c|c|c|c|c|c|}
\hline & $x^{11}$ & $x^{10}$ & $x^{9}$ & $x^{8}$ & $x^{7}$ \\
\hline$\alpha$ & - & - & - & - & - \\
\hline$\beta$ & + &,$- \frac{1}{2}$ & - & - & - \\
\hline$\alpha \beta$ & - &,$+ \frac{1}{2}$ & + & + & + \\
\hline
\end{tabular}

For model $\mathrm{B}^{\prime}$, there are two elements acting with fixed points. The element $\alpha$ is the same as before but $\beta$ differs from that of $\mathrm{A}^{\prime}$ in not having the shift in $x^{11}$. This makes it, in fact, the orbifold limit of $\left(K 3 \times S^{1}\right) / Z_{2}$ considered in Ref. [10], but we go through the analysis here again to show how it relates to the other cases. For $\alpha$, the twisted sector just gives the 165 -branes as before, which are again projected to half their number. But now the element $\beta$ also has fixed points. This time the twisted sector is that of IIA on $T^{4} / Z_{2}$, which in six-dimensional $N=1$ language has 16 vector and 16 hypermultiplets. Generically the gauge group is thus $U(1)^{16}$, which can be enhanced at special points upto $E_{8} \times E_{8}$. These have to be projected onto the sector invariant under the remaining generators. Once again, the action of $\alpha \beta$ permutes the vector multiplets pairwise, and so half of them will be invariant, leading to 8 vectors and 8 hypers. Putting everything together, one finds at generic points 9 tensors, 8 vectors and 20 hypermultiplets, the same as for F-theory on model B.

Model $\mathrm{C}^{\prime}: K 3 \times S^{1} / Z_{2}$

\begin{tabular}{|c|c|c|c|c|c|}
\hline & $x^{11}$ & $x^{10}$ & $x^{9}$ & $x^{8}$ & $x^{7}$ \\
\hline$\alpha$ & - & - & - & - & - \\
\hline$\beta$ & + & - & - & - & - \\
\hline$\alpha \beta$ & - & + & + & + & + \\
\hline
\end{tabular}

Finally, for model $\mathrm{C}^{\prime}$, the situation is more complicated. All the elements act with fixed points. In fact the action simply corresponds to $T^{4} / Z_{2} \times S^{1} / Z_{2}$. In this case, the 16 5 -branes are not permuted by the other symmetries. Rather, if they are located at their fixed points then the other actions leave them fixed. However, it can be argued that near any of the fixed points, four of the five transverse collective coordinates of the 5-brane get reversed by the action of the element $\beta$. As a result, the hypermultiplet degrees of freedom of each 5-brane get projected out and we are left with 16 tensor multiplets. This leaves the vector multiplets, which correspond to a rank 32 gauge group before projection (rank 16 from the twisted sector of $\beta$, which we have studied above, and another rank 16 from 
the twisted sector of $\alpha \beta$ which corresponds to M-theory on $S^{1} / Z_{2}$ (further compactified on $T^{4}$ ) and hence produces again the gauge sector of the heterotic string). This time it is easy to see that an abelian $U(1)^{16}$ gauge group is inconsistent with anomaly cancellation. Indeed, if $v$ and $h$ are the number of vector and hypermultiplets coming from these twisted sectors after projection, and $V, H$ and $T$ are the total numbers of vector, hyper and tensor multiplets in the model, then the anomaly cancellation condition

$$
H-V=273-29 T
$$

requires that we have

$$
h-v=-224
$$

This shows that we must have at least 224 vector multiplets, and moreover any additional vector multiplets will be accompanied by charged hypers. Thus the simplest solution is $v=224$ and $h=0$. This in turn can arise symmetrically only if we are at a point of enhanced symmetry with $S 0(8)^{8}$ gauge group, and the projection eliminates all the hypermultiplets that come along with the gauge multiplets. So we conclude that this orientifold gives a spectrum of 17 tensor, 224 vector and 4 hypermultiplets, precisely that of F-theory on model $\mathrm{C}$ at generic points.

Although the arguments in the last case seem less compelling than in the previous two, one can point out some intriguing properties in the M-theory picture. The two sets of vector multiplets that arise from the twisted sectors of $\beta$ and $\alpha \beta$ respectively, are believed to arise in M-theory by the wrapping respectively of a 5 -brane around directions $7,8,9,10$, and of a 2-brane around direction 11. If there is an electric-magnetic 2-brane-5-brane duality in M-theory, then it means that one $S 0(8)^{4}$ factor of the gauge group is electric-magnetic dual to the other, which might conceivably be checked once such a duality is better understood. In a heterotic picture this translates into perturbative and non-perturbative contributions of $S O(8)^{4}$ to the gauge group 30].

In studying the above model, we used the fact that the natural action of the element $\beta$ on the 5-brane multiplets was to reverse the sign of four of the five transverse brane coordinates. One may argue that it is equally natural to concentrate on $\gamma$ instead of $\beta$, in which case the natural action would be to reverse one of the five transverse brane coordinates. (In this case the element $\beta$ would have the opposite action to its natural one.) This will be consistent with supersymmetry only if the coordinate projected out is part of an $\mathrm{N}=1$ tensor multiplet, and if the accompanying tensor field also gets projected out. 
The result is then that we retain 16 hypermultiplets from the 5-brane sector, and project out all 16 tensor multiplets.

The corresponding model now has only one tensor multiplet (from the untwisted sector), and at least 20 hypers, of which 4 come from the untwisted sector and another 16 from the branes. The remaining states come from the twisted sectors of $\beta$ and $\gamma$. If we again denote by $h$ and $v$ the number of hyper and vector multiplets coming from these twisted sectors after projection, anomaly cancellation this time requires

$$
h-v=224
$$

The simplest solution of this arises by assuming that there are no vector multiplets at a generic point in moduli space, in which case we have $h=224$. The model therefore has a total of one tensor, no vectors and 244 neutral hypermultiplets at a generic point. This coincides with the generic spectrum of F-theory on the well-known (3,243) Calabi-Yau , dual to the $(12,12)$ embedding of heterotic string on K3. In fact, precisely $K 3 \times S^{1} / Z_{2}$ was considered in Ref. [30] to argue for heterotic-heterotic duality in this case.

It appears, therefore, that potentially there are two distinct versions of model $\mathrm{C}^{\prime}$. If this is the case, there must be two distinct ways of smoothing the orbifold $\mathrm{C}$ in $\mathrm{F}$ theory. Although we do not have a complete understanding of this situation, it appears to strongly resemble the phenomenon of discrete torsion 34] in Calabi-Yau compactifications of type II string theory. There, it is known [35] [36] 37 that there are two distinct theories associated to the orbifold that we have defined as model $\mathrm{C}$ above. The standard blowup, corresponding to deforming the Kähler structure, leads to the $(51,3)$ Calabi-Yau. This is the theory that one would have discovered following the usual rules for constructing orbifold compactifications in string theory, since the twisted sector modes that string theory produces are exactly the marginal deformations of Kähler structure required to smooth the orbifold to the $(51,3)$ Calabi-Yau.

However, one can instead consistently require that the elements $\beta$ and $\gamma$ of the orbifolding group act with an extra minus sign on twisted-sector states of $\alpha$, and similarly for the other two twisted sectors. This choice was interpreted in Refs. 35] 36] as arising from the presence of "discrete torsion". (A more detailed analysis of this issue in terms of Brauer groups appeared in Ref. [37].) In this case, one finds that the twisted sector states correspond to deformations of the complex, rather than Kähler, structure, and the orbifold gets deformed to a (singular) Calabi-Yau manifold which still retains 64 nodes, with Hodge numbers $(3,51)$. 
It should be evident that the two versions of model $\mathrm{C}^{\prime}$ that we have discovered (which we denote henceforth by $\mathrm{C}^{\prime}(1)$ and $\mathrm{C}^{\prime}(2)$ ) have a lot in common with the discrete torsion problem discussed above: in the twisted sector of $\alpha$, model $\mathrm{C}^{\prime}(1)$ keeps 16 tensor multiplets and projects out 16 hypers, while model $\mathrm{C}^{\prime}(2)$ does the opposite. Similarly, in the twisted sectors for $\beta$ and $\gamma$, model $\mathrm{C}^{\prime}(1)$ keeps a total of 224 vector multiplets, which give rise to the gauge group $S O(8)^{8}$, while model $\mathrm{C}^{\prime}(2)$ keeps instead 224 hypermultiplets, which are of course neutral as there is no gauge group. Thus complementary sets of multiplets are projected in/out. The somewhat surprising conclusion is that the analogue of discrete torsion in M-theory (and, as we argue below, in F-theory) relates two smooth CalabiYau's with Hodge numbers $(51,3)$ and $(3,243)$, rather than relating the smooth $(51,3)$ to its "mirror", the $(3,51)$ with 64 nodes.

Let us briefly examine how the same ambiguity arises in F-theory on model C. Here, the twisted sector for $\beta$ produces 24 F-theory 7-branes, of which 6 are located at each of the 4 fixed points of $\beta$ on the $\left(x^{7}, x^{8}\right)$ torus, and all are wrapped around the $\left(x^{9}, x^{10}\right)$ torus. The twisted sector for $\alpha \beta$ is analogous, with the roles of $\left(x^{7}, x^{8}\right)$ and $\left(x^{9}, x^{10}\right)$ interchanged. Finally, the twisted sector for $\alpha$ just consists of the usual twisted-sector states of type IIB on $\mathrm{K} 3$, which are well-known to correspond to 16 tensor multiplets of $\mathrm{N}=2$, equivalent to 16 tensor and 16 hypermultiplets of $\mathrm{N}=1$. The projection leading to F-theory on $(51,3)$ keeps all the $\mathrm{N}=1$ tensor multiplets, while that leading to F-theory on $(3,243)$ keeps the hypermultiplets. We will not discuss the 7-brane sectors here since they are known to be quite subtle in F-theory[2], but the observations above should be sufficient to see that the discrete-torsion-like phenomenon, of projecting in/out complementary sets of states, operates in F-theory too. It is amusing to note a different kind of complementarity between M- and F-theory in this model (and the other ones discussed above): multiplets supported on branes in either description are "fundamental" states in the other one.

\section{Mappings between M-theory, F-theory and IIB orientifolds}

Having discussed three pairs of candidate M-theory-F-theory duals, we will now demonstrate the proposed equivalence as also the relation to some familiar and unfamiliar IIB orientifolds. The strategy will be to map the symmetries of one theory to the other at the orbifold point. Since the spectra are independently known to coincide, identifying the map between symmetries automatically implies that orbifolding commutes with duality. (The conditions under which orbifolding commutes with duality have been recently 
examined in a variety of situations[7]. Our case is of the type where this is known to hold in a variety of cases.)

We first demonstrate the equivalence upon compactification to 5 dimensions. Since F-theory on $X \times S^{1}$ is on the same moduli space as M-theory on $X$, this has the advantage of reducing the problem to that between two different M-theory compactifications. But we will, in a more direct manner, also be able to show the equivalence in $6 \mathrm{~d}$ by mapping the orbifolding symmetries in F-theory to those of IIB. This will also make transparent the relation to the IIB orientifold.

Thus, to start with, we compactify the models of Section 2 on a circle, so that the question reduces to duality between M-theory on the Calabi-Yau's given by models A,B and $\mathrm{C}$ above, and type IIA on the corresponding orientifolds $\mathrm{A}^{\prime}, \mathrm{B}^{\prime}$ and $\mathrm{C}^{\prime}$. Let us focus on models $\mathrm{C}$, the $(51,3)$, and $\mathrm{C}^{\prime}$ for definiteness and simplicity. Since we have compactified to one lower dimension, the coordinate labellings for the various orbifold actions will all have to be shifted down by 1 unit with respect to those displayed in the tables.

The symmetries of M-theory can be mapped to those of type IIA as follows: An inversion in the 11 th direction is mapped to $(-1)^{F_{L}}$ where $F_{L}$ is the spacetime fermion number for left-moving states on the worldsheet [7]. Also an inversion of an odd number of spatial dimensions in type IIA must be accompanied by a world-sheet orientation reversal, $\Omega$, to be a symmetry. We can now easily read off from table $\mathrm{C}$ that M-theory on the $(51,3)$ can be written as an orbifold of type IIA on $T^{5}$ modded out by $\left\{1, I_{6789}, \Omega(-1)^{F_{L}} I_{6710}\right.$, $\left.\Omega(-1)^{F_{L}} I_{8910}\right\}$. Here $I_{\ldots}$ represents inversions in the relevant directions.

For future use, we list some of the relevant properties of the symbols that we will use. The square of an inversion acts as \pm 1 on spacetime fermions according to

$$
\begin{aligned}
I_{n_{1} \ldots n_{k}}^{2} & =1, \quad k=0,1 \bmod 4 \\
& =(-1)^{F_{L}+F_{R}}, \quad k=2,3 \bmod 4
\end{aligned}
$$

as one can easily show using $\Gamma$-matrices. Orientation reversal does not commute with reversing the sign of left-moving fermions, and in fact we get

$$
\begin{aligned}
\Omega(-1)^{F_{L}} \Omega & =(-1)^{F_{R}} \\
\left(\Omega(-1)^{F_{L}}\right)^{2} & =(-1)^{F_{L}+F_{R}}
\end{aligned}
$$

From these facts one can check that all elements of the action described above are of order two as they should be. 
Some additional results that we will need state that (at least for orbifold groups involving only $Z_{2}$ factors) S-duality of type IIB interchanges $\Omega$ and $(-1)^{F_{L}}$, that under T-duality on all $k$ directions $\left(T_{n_{1} \ldots n_{k}}\right)$ the inversion $I_{n_{1} \ldots n_{k}}$ goes to $(-1)^{k F_{L}} I_{n_{1} \ldots n_{k}}$, and that under T-duality the orientation-reversal $\Omega$ picks up an inversion in all the dualized directions, along with a possible factor of $(-1)^{F_{L}}$ which can be most simply determined by requiring that all elements be of order 2 .

Now, we have the following chain of equivalences:

$$
\begin{aligned}
& \text { IIA on } T^{5} /\left\{1, I_{6789}, \Omega(-1)^{F_{L}} I_{6710}, \Omega(-1)^{F_{L}} I_{8910}\right\} \\
& \stackrel{T_{8}}{\longrightarrow} \text { IIB on } T^{5} /\left\{1,(-1)^{F_{L}} I_{6789}, \Omega I_{67810}, \Omega(-1)^{F_{L}} I_{910}\right\} \stackrel{S}{\longrightarrow} \text { IIB on } T^{5} /\left\{1, \Omega I_{6789},(-1)^{F_{L}} I_{67810}, \Omega(-1)^{F_{L}} I_{910}\right\} \\
& \stackrel{T_{10}}{\longrightarrow} \text { IIA on } T^{5} /\left\{1, \Omega I_{678910}, I_{67810}, \Omega I_{9}\right\} \\
& \stackrel{9 \leftrightarrow 10}{\longrightarrow} \text { IIA on } T^{5} /\left\{1, \Omega I_{678910}, I_{6789}, \Omega I_{10}\right\}
\end{aligned}
$$

Comparison of the last line with table $\mathrm{C}^{\prime}$ reveals that it corresponds precisely to type IIA on the orientifold model $\mathrm{C}^{\prime}$. The same manipulations can be used to prove the equivalence of M-theory on the Calabi-Yau's $(11,11)$ (model A) and $(19,19)$ (model B) to type IIA on the orientifolds $T^{5} /\left(Z_{2} \times Z_{2}\right)\left(\mathrm{A}^{\prime}\right)$ and $\left(K 3 \times S^{1}\right) / Z_{2}\left(\mathrm{~B}^{\prime}\right)$ respectively, the only difference being that one has to incorporate the relevant shifts. Since these act in directions along which we have not T-dualized in the above chain, they go through unaffected.

Thus, at least after compactifying to 5 dimensions, the proposed equivalence of $\mathrm{F}$ theory on orbifolds and M-theory on orientifolds can be demonstrated, not just by matching spectra, but by directly identifying symmetries of the two models, and orbifolding by the corresponding symmetries on each side. This may perhaps be enough to prove equivalence in 6 dimensions, for the following reason. In the $(11,11)$ case, since there are no vector fields in the $6 \mathrm{~d}$ spectrum, the $5 \mathrm{~d}$ equivalence which relates the vectors to vectors must come from a $6 \mathrm{~d}$ equivalence in which the tensors are mapped to tensors. The map between hypermultiplets is the same in 5 and 6 dimensions. Thus there is no ambiguity in going back to 6 from 5 dimensions in this case. For the $(19,19)$, in 5 dimensions we have 18 vector multiplets. Discounting the one coming from compactification and one more coming from an untwisted-sector tensor in $6 \mathrm{~d}$, the remaining 16 come from 8 vectors and 8 tensors in $6 \mathrm{~d}$. Since they are on an equal footing in $5 \mathrm{~d}$, one might wonder if the map between them actually corresponds to an exchange among tensors and vectors in 6d, in which case $5 \mathrm{~d}$ equivalence would not lift to one in $6 \mathrm{~d}$. That this does not happen can be easily argued. 
The fields in the $(11,11)$ example are a proper subset of those in $(19,19)$, and the equivalence in the former (between $\mathrm{A}$ and $\mathrm{A}^{\prime}$ ) lifts, as we have just seen, to an equivalence in $6 \mathrm{~d}$. This means that the tensors in the (19.19) example (B and $\left.\mathrm{B}^{\prime}\right)$ do get identified with each other under our dualities, and there is no possibility of an interchange with vectors. While this chain of arguments supports the equivalence in 6 dimensions, a more direct demonstration will be given below.

It is perhaps worthwhile to stress that the equivalence we have demonstrated, between F-theory on orbifolds and M-theory on orientifolds, is completely different from the wellknown relation betwen F-theory on an orbifold (or any elliptically fibred manifold) and M-theory on the same orbifold or manifold, which leads to a theory in one lower dimension.

Continuing on the above lines, one can also, in 5 dimensions, map the models of interest to orientifolds of type IIB. Performing a T-duality in the 10 direction on the last line of Eq.(3.3), we find that the $(51,3)$ case (models $\mathrm{C}$ and $\mathrm{C}^{\prime}$ ) is also equivalent to type IIB on the orientifold $T^{5} /\left\{1, I_{6789}, \Omega, \Omega I_{6789}\right\}$ which at first sight is the circle compactification of the orientifold studied by Gimon and Polchinski[18]. We will explain, after a direct demonstration of the equivalence in $6 \mathrm{~d}$, why this is not the case.

We have seen that conjectured F-theory, M-theory and IIB orientifold duals can be identified by a sequence of $\mathrm{T}$ and S-dualities in 5 dimensions. One may ask if the identifications can be demonstrated directly in 6 dimensions. Since F-theory has no S-duality, all dualities having been converted to T-dualities, a chain of equivalences in $6 \mathrm{~d}$ is likely to be simpler and more direct. For this, we need to map the orbifolding symmetries of F-theory directly to those of type IIB. Just as for M-theory, an inversion in the 11 direction was identified to the operator $(-1)^{F_{L}}$ in type IIA, for F-theory a pair of inversions in the 11,12 directions can be identified with the product $\Omega(-1)^{F_{L}}$ in type IIB[16]. This follows again by carefully examining the action of these inversions on the states of type IIB. One support for this identification comes from the fact that, for orbifold groups which are powers of $Z_{2}$, S-duality in type IIB exchanges $\Omega$ and $(-1)^{F_{L}}$, and in F-theory this is manifested as the exchange of the 11 and 12 directions.

With this addition to the previous rules, we find directly that F-theory on the $(51,3)$ (model C) to 6 dimensions can be written as type IIB on $T^{4} /\left\{1, I_{78910}, \Omega(-1)^{F_{L}} I_{78}\right.$, $\left.\Omega(-1)^{F_{L}} I_{910}\right\}$. We now have a short chain of equivalences:

$$
\begin{aligned}
& \text { IIB on } T^{4} /\left\{1, I_{78910}, \Omega(-1)^{F_{L}} I_{78}, \Omega(-1)^{F_{L}} I_{910}\right\} \\
\stackrel{T_{7}}{\longrightarrow} & \text { IIA on } T^{4} /\left\{1,(-1)^{F_{L}} I_{78910}, \Omega(-1)^{F_{L}} I_{8}, \Omega I_{7910}\right\} \\
\stackrel{T_{8}}{\longrightarrow} & \text { IIB on } T^{4} /\left\{1, I_{78910}, \Omega, \Omega I_{78910}\right\}
\end{aligned}
$$


The middle line can be recognised precisely to be the M-theory orientifold on model $\mathrm{C}^{\prime}$ after using the lorentz invariance among the 11 directions to interchange $x^{11}$ and $x^{8}$. This is in consonance with the fact that we would not expect the M-theory orientifold to be perturbatively T-dual to the F-theory compactification. Interchanging $x^{11}$ and $x^{8}$ is a non-perturbative operation in string theory. The last line, which is the result of T-dualizing the F-theory on directions 9 and 10 simultaneously, leads once again (apparently) to the Gimon-Polchinski model. But clearly the GP model spectrum (with 1 tensor) does not agree with that of F-theory on the (51,3) (with 17 tensors).

Here we find support for the idea, proposed in the previous section, that there are actually two distinct versions of model C (in F-theory) or model $\mathrm{C}^{\prime}$ (in M-theory). It is now understood[23] that the Gimon-Polchinski model is equivalent to an F-theory compactification on the $(3,243)$ Calabi-Yau, which in turn is known[31] to be dual to the $E_{8} \times E_{8}$ heterotic string on $\mathrm{K} 3$ with the symmetric $(12,12)$ embedding of instanton numbers in the two $E_{8}$ factors. Thus, on relating our $\mathrm{C}$ and $\mathrm{C}^{\prime}$ models to orientifolds, we have apparently found a second realisation of these models.

However, it has recently been noticed[24] that there is actually an ambiguity in the action of the orientation-reversal operator $\Omega$ on twisted-sector states. The model is completely characterised only after this action is specified. We expect that the twofold ambiguity that we discovered above, in models $\mathrm{C}$ and $\mathrm{C}^{\prime}$, will be mirrored in the orientifold construction. Both models should have the common feature of having both 5- and 9-branes. This is easily seen to arise on T-duality from the two sets of 7-branes which are wrapped around $x^{7}, x^{8}$ and $x^{9}, x^{10}$ respectively.

Continuing with the original discussion on the relation to orientifolds, we note that the same short chain maps the $(19,19)$ (models B, $\left.\mathrm{B}^{\prime}\right)$ first to M-theory on $\left(K 3 \times S^{1}\right) / Z_{2}$ and then to the orientifold IIB on $T^{4} /\left\{1, I_{78910}, \Omega I_{78910} S_{10}, \Omega S_{10}\right\}$ studied in Ref. [19]. (Here, $S_{10}$ is a shift by a half unit in the 10 direction.) As another consistency in this mapping we note that we originally had 7 -branes wrapped around $x^{7}, x^{8}$ in the F-theory. T-dualising in these directions gives us the 5-branes of Ref. [19] distributed in exactly the way one obtains $S O(8)^{2}$ enhanced gauge group with hypermultiplets in the adjoint in their picture. We also see that there are no 9-branes, as expected.

For the $(11,11)$ (models $\left.\mathrm{A}, \mathrm{A}^{\prime}\right)$ the situation is slightly different. There, the analogue of the first line in Eq.(3.4) is type IIB on $T^{4} /\left\{1, I_{78910}, \Omega(-1)^{F_{L}} I_{78} S_{8} S_{10}\right.$, $\left.\Omega(-1)^{F_{L}} I_{910} S_{8} S_{10}\right\}$. M-theory on $\mathrm{A}^{\prime}$ follows as usual after a $T_{7}$. But this time, we have a shift in the direction 8 where we want to perform a T-duality. Under $T_{78}$, the shift $S_{8}$ 
will be replaced by a "winding shift" $\hat{S}_{8}$ and so we will find the orientifold of type IIB

on $T^{4} /\left\{1, I_{78910}, \Omega \hat{S}_{8} S_{10}, \Omega I_{78910} \hat{S}_{8} S_{10}\right\}$. This appears to be a new orientifold of type IIB. Note that since there were no 7-branes in the F-theory, there are neither 5-branes nor 9-branes in the T-dual theory. This feature, reflecting no net tadpole in the one loop worldsheet contributions, was also present in the $T^{4} / Z_{4}$ orbifold of 20], 21].

The success of these manipulations encourages us to investigate the case of F-theory compactifications on 8-dimensional orbifolds down to $4 \mathrm{~d}$, and relate them to orientifolds of M-theory on $T^{7} /\left(Z_{2}\right)^{3}$, obtaining $\mathrm{N}=1$ supersymmetric theories in 4 dimensions. We will also find realizations of these models as type IIB orientifolds on $T^{6}$. A larger class of models arises by starting with non-toroidal orbifolds in F-theory, of the form $\left(K 3 \times T^{4}\right) /\left(Z_{2}\right)^{2}$, which we identify with M-theory on seven-dimensional orientifolds $\left(K 3 \times T^{3}\right) /\left(Z_{2}\right)^{2}$ and IIB on six-dimensional orientifolds $\left(K 3 \times T^{2}\right) /\left(Z_{2}\right)^{2}$.

\section{Compactifications to four dimensions}

The Calabi-Yau threefolds A, B and C that we have discussed are special cases of the so-called Voisin-Borcea models, all of which are defined by taking $\left(K 3 \times T^{2}\right) / Z_{2}$, where the $Z_{2}$ acts as reflection on the 2-torus and as an involution[38] on K3 which reverses the sign of the $(2,0)$ form. The possible such involutions have been classified and are labelled by three integers $(r, a, \delta)$. (For a table of the allowed cases, see for example Ref.[15].) One might also imagine constructing a large class of Calabi-Yau 4-folds of the form $(K 3 \times K 3) / Z_{2}$ using these involutions. Indeed, Calabi-Yau 4-folds of this form with $S U(4)$ holonomy have been considered by Borcea[27, with the $Z_{2}$ action on the K3's labelled by $\left(r_{1}, a_{1}, \delta_{1} ; r_{2}, a_{2}, \delta_{2}\right)$. These involutions reverse the individual $(2,0)$ forms while preserving the $(4,0)$ form. These therefore provide a large class of $N=1$ vacua in four dimensions when utilised for F-theory compactifications.

The A,B and C threefolds that we studied in previous sections corresponded to choosing the three involutions on $K 3$ that can be defined in a toroidal orbifold limit. (By abuse of notation, we label the involutions $\mathrm{A}, \mathrm{B}$ and $\mathrm{C}$ as well.) In terms of $(r, a, \delta)$ they are $(10,10,0),(10,8,0)$ and $(18,4,0)$ respectively.) For fourfolds, there is a variety of orbifold limits that one can discuss. We first consider a couple of families which realise a $\left(T^{4} / Z_{2} \times K 3\right) / Z_{2}$ limit and later, some special cases admitting a $T^{8} /\left(Z_{2}\right)^{3}$ orbifold point.

In the former category, let us start with the $Z_{2}$ acting as a $(C, \sigma)$ involution on the $T^{4} / Z_{2}$ and $K 3$ respectively. Here $\sigma \equiv \sigma(r, a, \delta)$ is a general $K 3$ involution reversing the 
$(2,0)$ form. Choosing the fibre for F-theory compactification in the $T^{4}$ (with coordinates $x^{9} \ldots x^{12}$; the $K 3$ will be labelled by $x^{5} \ldots x^{8}$ ) the action of the $Z_{2}$ 's can be summarised as

Model $(\mathrm{C}, \sigma)$ :

\begin{tabular}{|c|c|c|c|c|c|}
\hline & $x^{12}$ & $x^{11}$ & $x^{10}$ & $x^{9}$ & $x^{8} \ldots x^{5}$ \\
\hline$\alpha$ & - & - & - & - & + \\
\hline$\beta$ & + & + & - & - & $\sigma$ \\
\hline$\alpha \beta$ & - & - & + & + & $\sigma$ \\
\hline
\end{tabular}

The Hodge numbers and Euler characteristics of this family of 4-folds can be worked out using the general expression in Ref. 27], giving

$$
\begin{aligned}
& H^{1,1}=26+5 r-4 a, \quad H^{2,1}=88-4 r-4 a \\
& H^{3,1}=22-r, \quad H^{2,2}=60+24 r-8 a \\
& \chi=2\left(2+H^{1,1}+H^{3,1}-2 H^{2,1}\right)+H^{2,2}=48(r-4)
\end{aligned}
$$

We note that the Euler characteristic $\chi$ is a multiple of 24, as expected[25], and is negative for $0 \leq r \leq 3$. Thus, these cases are likely to lead to F-theory compactifications which are inconsistent because of tadpoles [25]. For $r=4$ we have no tadpoles, while for $r>4$ the tadpoles can be cancelled by assuming condensation of $2(r-4)$ IIB 3-branes in the vacuum.

The equivalence to M-theory follows as before: The above orbifold can be written as type IIB on $\left(T^{2} \times K 3\right) /\left\{1, \Omega(-1)^{F_{L}} I_{910}, I_{910} \sigma, \Omega(-1)^{F_{L}} \sigma\right\}$. Our short chain now reads:

$$
\begin{aligned}
\text { IIB on }\left(T^{2} \times K 3\right) /\left\{1, \Omega(-1)^{F_{L}} I_{910}, I_{910} \sigma, \Omega(-1)^{F_{L}} \sigma\right\} \\
\stackrel{T_{9}}{\longrightarrow} \text { IIA on }\left(T^{2} \times K 3\right) /\left\{1, \Omega I_{10},(-1)^{F_{L}} I_{910} \sigma, \Omega(-1)^{F_{L}} I_{9} \sigma\right\} \\
\stackrel{T_{10}}{\longrightarrow} \text { IIB on }\left(T^{2} \times K 3\right) /\left\{1, \Omega, I_{910} \sigma, \Omega I_{910} \sigma\right\}
\end{aligned}
$$

The middle line, after interchanging $x^{11}$ and $x^{10}$, is precisely M-theory on the orbifold $S^{1} / Z_{2} \times\left(T^{2} \times K 3\right) /\left\{1, I_{910} \sigma\right\}$. Note that $\left(T^{2} \times K 3\right) /\left\{1, I_{910} \sigma\right\}$ are the Voisin-Borcea 3-folds $C Y_{\sigma}$ with Hodge numbers

$$
H^{1,1}=5+3 r-2 a, \quad H^{2,1}=65-3 r-2 a
$$


The last line of (4.2) is the orientifold of type IIB on $C Y_{\sigma} /\{1, \Omega\}$, which at first sight seems to be the same as Type I on $C Y_{\sigma}$. The subtleties that arose in the $6 \mathrm{~d}$ examples recur, however, in this case as well, and we will have more to say on this below.

What can one say about the spectrum of these models? Unfortunately, F-theory on 4 -folds has not so far been studied in great detail. So we restrict ourselves to some naive deductions. We can learn something about the gauge group by looking at the singularity of the fibre. The fibre degenerates to $T^{2} / Z_{2}$ over certain fixed divisors in the base. These consist of the following: The element $\alpha$ has 4 copies of $K 3 / \sigma$ as its fixed locus. The element $\gamma$ also has degenerating fibres over the fixed loci of $\sigma$ in K3. These latter are known to consist of $(k+1)$ components (see for instance Ref. 115]) where $k=\frac{1}{2}(r-a)$. Of these, $k$ are rational curves and 1 is of genus $g=\frac{1}{2}(22-r-a)$ Since each component contributes an $S O(8)$ (at least naively), one has apparently an $S O(8)^{k+5}$ gauge group. An exception is the $(C, A)$ case, since the Enriques involution $A$ has no fixed points and therefore the group is $S O(8)^{4}$. This also seems consistent with the following fact: assuming no $U(1)$ factors, we must have

$$
r(V)=H^{1,1}(X)-H^{1,1}(B)-1
$$

Examining the table for $(C, \sigma)$, we see that $H^{1,1}(B)=H^{1,1}\left(C Y_{\sigma}\right)$. Using (4.3) and (4.1) we obtain $r(V)=4(k+5)$ as desired. In the IIB orientifold, these vector multiplets come from the open string twisted sector states.

The closed string sectors (twisted and untwisted), of the orientifold give rise to some neutral chiral multiplets. These are easy to count. They are simply the closed string states of Type I on $C Y_{\sigma}$ and are $H^{1,1}+H^{2,1}+1$ in number. (The 1 counts the dilaton chiral multiplet.) In the case of our 3-folds, Eqn.(4.3) determines this number to be $71-4 a$.

Finally, there can be charged chiral multiplets as well. These are determined by the nature of the fixed divisor on the base. We will assume that the only charged matter comes from the divisor containing the genus $g$ curve. This was also the source of charged matter in 6 dimensions. Then there are $g$ chiral multiplets in the adjoint of the $S O(8)$ associated to this divisor. Thus we have $24 g$ multiplets charged under the gauge group, and $4 g$ which are neutral. We will show that this spectrum satisfies a non-trivial consistency requirement.

Consider M-theory compactified on the same 4-fold down to 3 spacetime dimensions. The number of neutral multiplets in $3 \mathrm{~d}$ (where vectors are dual to scalars) is $H^{1,1}+H^{2,1}+$ 
$H^{3,1}$. Thus one has the following constraint from compactification of our original theory on a circle where the gauge group is Higgsed:

$$
r(V)+c^{0}+1=H^{1,1}+H^{2,1}+H^{3,1}
$$

where $c^{0}$ is the number of neutral chiral multiplets, and the 1 comes from the gravity multiplet. Substituting $r(V)=4(k+5)=2 r-2 a-20$ and $c^{0}=4 g+(71-4 a)=115-2 r-6 a$, together with the hodge numbers from (4.1), we see that Eqn.(4.5) is satisfied. This gives us confidence that our assumptions were well-founded. There can be extra contributions from the $\chi / 24=2 r-83$-branes of IIB necessary to cancel the tadpole. These will be invisible in (4.5) since the M-theory compactification would also have extra membranes.

One can similarly define another family of fourfolds admitting $\left(T^{4} / Z_{2} \times K 3\right) / Z_{2}$ orbifold limits. Here the $Z_{2}$ acts as a $(B, \sigma)$ involution and one can consider F-theory compactification on

Model $(\mathrm{B}, \sigma)$ :

\begin{tabular}{|c|c|c|c|c|c|}
\hline & $x^{12}$ & $x^{11}$ & $x^{10}$ & $x^{9}$ & $x^{8} \ldots x^{5}$ \\
\hline$\alpha$ & - & - & - & - & + \\
\hline$\beta$ & + & + &,$- \frac{1}{2}$ & - & $\sigma$ \\
\hline$\alpha \beta$ & - & - &,$+ \frac{1}{2}$ & + & $\sigma$ \\
\hline
\end{tabular}

This time the Hodge numbers are

$$
\begin{array}{ll}
H^{1,1}=12+2 r-a, & H^{2,1}=24-2 a \\
H^{3,1}=52-2 r-a, & H^{2,2}=252-4 a \\
\chi=288 &
\end{array}
$$

The same manipulations as in (4.2) now give us M-theory on the orbifold $\left(S^{1} / Z_{2} \times\right.$ $\left.T^{2} \times K 3\right) / Z_{2}$ where the second $Z_{2}$ acts as a pure shift $+\frac{1}{2}$ on the $S^{1}$ and the usual $\left\{I_{910} \sigma\right\}$ on the $T^{2} \times K 3$. However, after another T-duality we end up with a IIB orientifold with a 'winding shift'.

The gauge group in this case is $S O(8)^{2}$ at the orbifold point, independent of $\sigma$, for exactly the same reasons as in the $6 \mathrm{~d}$ model B: $(19,19)$. The charged matter in this case consists of 2 adjoints of $S O(8)$ which Higgs the group at a generic point giving $U(1)^{8}$ together with 8 chiral multiplets. More neutral chiral multiplets come, as before from the closed string sector states of the orientifold, which once again turn out to be 
$71-4 a$ in number. It is again a non-trivial check to see that (4.5) is satisfied. Substituting $r(V)=8, c^{0}=8+(71-4 a)$ and the hodge numbers from (4.6), we once again match the expressions.

The number of 3 -branes needed to cancel the 2 form tadpole is always $\chi / 24=12$. We also note in passing that F-theory on the $(C, B)$ orbifold is not the same as on the $(B, C)$ — they have different gauge groups, $S O(8)^{6}$ and $S O(8)^{2}$ respectively. Thus it does matter whether we choose our fibre to have either the $C$ or $B$ involution, and reflects a lack of 12 dimensional Lorentz invariance.

F-theory compactifications on many other Borcea 4-folds can also be considered, and we reserve this for future study. For instance, another interesting family is the $(A, \sigma)$ all of whose members have $\chi=288$. We proceed instead to examine a few special cases, some of which are members of the two families described above. These are the cases where one can go to the $T^{8} /\left(Z_{2}\right)^{3}$ point in moduli space.

\section{Some Particular Four-dimensional Compactifications}

The $T^{8} /\left(Z_{2}\right)^{3}$ orbifold point of $(K 3 \times K 3) / Z_{2}$ is realised in 6 distinct fourfolds labelled by their involutions - (A,A), (B,B), (C,C), (A,B), (A,C) and $(\mathrm{B}, \mathrm{C})$. We note once again that each of these manifolds can realise a number of different F-theory vacua depending on the choice of fibre.

Since it is tedious to list all 7 nontrivial elements of $\left(Z_{2}\right)^{3}$, in this section we will often list only the three generators $\alpha, \beta$ and $\gamma$, one for each $Z_{2}$ factor. It must be kept in mind that, depending on the shifts included, the other elements that we do not always write explicitly may or may not have fixed points.

Let us focus on the (C,C) fourfold, which has no shifts. This is, of course, a member of the family studied in the previous section with $\sigma=C=(18,4,0)$ and is the $4 \mathrm{~d}$ analogue of the threefold $(51,3)$. Its generators are as follows:

Model (C,C):

\begin{tabular}{|c|c|c|c|c|c|c|c|c|}
\hline & $x^{12}$ & $x^{11}$ & $x^{10}$ & $x^{9}$ & $x^{8}$ & $x^{7}$ & $x^{6}$ & $x^{5}$ \\
\hline$\alpha$ & + & + & + & + & - & - & - & - \\
\hline$\beta$ & - & - & - & - & + & + & + & + \\
\hline$\gamma$ & - & - & + & + & - & - & + & + \\
\hline
\end{tabular}


This corresponds to type IIB on the orientifold $T^{6} /\left\{1, I_{5678}, \Omega(-1)^{F_{L}} I_{910}, \Omega(-1)^{F_{L}} I_{78}\right\}$. (Recall that here we are listing only the generators and not all the nontrivial elements.) By a T-duality in the 9 direction we get IIA on $T^{6} /\left\{1, I_{5678}, \Omega I_{10}, \Omega(-1)^{F_{L}} I_{789}\right\}$ which is the same, after interchange of $x^{10}$ and $x^{11}$, as the M-theory orientifold $T^{7} /\left(Z_{2}\right)^{3}=$ $S^{1} / Z_{2} \times T^{6} /\left(Z_{2}\right)^{2}$ which we label $\left(\mathrm{C}^{\prime}, \mathrm{C}^{\prime}\right)$ :

Model $\left(\mathrm{C}^{\prime}, \mathrm{C}^{\prime}\right)$ :

\begin{tabular}{|c|c|c|c|c|c|c|c|}
\hline & $x^{11}$ & $x^{10}$ & $x^{9}$ & $x^{8}$ & $x^{7}$ & $x^{6}$ & $x^{5}$ \\
\hline$\alpha$ & + & + & + & - & - & - & - \\
\hline$\beta$ & - & + & + & + & + & + & + \\
\hline$\gamma$ & + & - & - & - & - & + & + \\
\hline
\end{tabular}

Applying, instead, a T-duality in the pair of directions 9,10 we find, after a change of generators, an orientifold of type IIB on $T^{6} /\left\{1, \Omega, I_{5678}, I_{78910}\right\}$ which is, in appearance, the same as the model studied in Ref.[22].

Let us look at the spectrum of this model. The Hodge numbers and Euler characteristic of the fourfold are given by

$$
\begin{aligned}
\left(H^{1,1}, H^{2,1}, H^{3,1}, H^{2,2}\right) & =(100,0,4,460) \\
\chi & =672
\end{aligned}
$$

From the results of the previous section we know the following. The gauge group is $S O(8)^{12}$, corresponding to $k=7$, which can also be argued as in the case of the $(51,3)$. Since $g=0$ in this case, there are apparently no charged hypermultiplets to Higgs the gauge group. The number of neutral chiral multiplets, $71-4 a$ is therefore 55 , which is also the number of chiral multiplets obtained in the closed string sector (twisted and untwisted) in [22]. However, the open string spectrum is different from theirs. This is once again due to the ambiguity that we encountered in the $(51,3)$ case. We expect that an orientifold with the complementary action of $\Omega$ on twisted sector states will realise the spectrum that we find here. There is no contradiction between this and the fact that the closed string twisted sector states of the two theories agree. In fact, the closed string twisted sector states are in $N=2$ hypermultiplets (each of which decomposes to two $N=1$ chiral multiplets) before the $\Omega$ projection. We therefore see that both actions of $\Omega$, in this sector, will produce chiral multiplets.

This can be understood geometrically in F-theory, where we can immediately identify two distinct Calabi-Yau manifolds to which this orbifold can be smoothened. Changing the 
Kähler structure (by blow-ups) leads to the Borcea 4-fold which we have been discussing. The other way to go away from the orbifold limit is to think of the singular base $T^{6} /\left(Z_{2}\right)^{3}$, which is actually $\left(T^{2} / Z_{2}\right)^{3}$, as a limit of $\left(P^{1}\right)^{3}$ as one varies the complex structure. Thus, it is the limit of an elliptic fibration of the form

$$
y^{2}=x^{3}+f(u, v, w) x+g(u, v, w)
$$

where $u, v, w$ are the inhomogeneous coordinates on $\left(P^{1}\right)^{3}$ and $(f, g)$ are polynomials of degrees $(8,12)$ respectively, in all their arguments. It is easy to see that this manifold will have $H^{1,1}=4$ and $H^{3,1}=2916$, the latter number arises as $(9)^{3}+(13)^{3}-3-3-3-1$ from the polynomial deformations in Eq.(5.2) above.

This smooth manifold, fibred over a $\left(P^{1}\right)^{3}$ base, is the strict analogue of the $(3,243)$ Calabi-Yau 3-fold that has been studied in the context of F-theory compactification to 6 dimensions. Thus, we claim that the $Z_{2} \times Z_{2}$ orientifold of type IIB on $T^{6}$ studied in Ref. [22] is dual to F-theory on the above elliptically fibred Calabi-Yau 4-fold. The relation between the two follows from a rerun of the arguments above at the orbifold point. We note that this manifold posseses many interesting features, such as the symmetry under the interchange of the $P^{1}$ 's. The corresponding exchange symmetry had reflected itself in the heterotic dual of the $(3,243)$ case in 6 dimensions as strong-weak duality. We expect a similar effect here, leading to a heterotic-heterotic dual family in $4 \mathrm{~d}$ related by the permutation group $S_{3}$.

It will be interesting to see if there are still other distinct ways to smoothen this orbifold, since after all 4-folds have other deformations besides those of Kähler and complex structure. Indeed, there are actually 4 ways to project out an $\mathrm{N}=1$ multiplet from an $\mathrm{N}=4$ multiplet since the latter, which is necessarily a vector multiplet, decomposes into one vector and three chiral multiplets of $\mathrm{N}=1$. This is in tantalising correspondence with the fact that a Calabi-Yau 4-fold has 4 independent Hodge numbers, $\left(H^{1,1}, H^{2,1}, H^{3,1}, H^{2,2}\right)$. Also notice that the discrete torsion for an orbifold group of the form $\left(Z_{2}\right)^{3}$ takes values in $Z_{2} \times Z_{2}$, which is of order four.

An argument that we will outline in the case of M-theory indicates that we will in general encounter the full discrete torsion. The fourfold ambiguity is also present in the IIB orientifold on $T^{6} /\left(Z_{2}\right)^{2}$ since in addition to the ambiguity in $\Omega$ one could also introduce the conventional discrete torsion in the conformal field theory.

Now let us say a few words about the $\left(\mathrm{C}^{\prime}, \mathrm{C}^{\prime}\right)$ compactification of M-theory. Of the 7 nontrivial elements of the $\left(Z_{2}\right)^{3}$ orbifolding group, 1 element $(\beta)$ corresponds to inversion 
of a single space coordinate, 3 elements $(\alpha, \gamma$ and $\alpha \gamma)$ correspond to inversions of 4 space coordinates, and the remaining $3(\alpha \beta, \beta \gamma$ and $\alpha \beta \gamma)$ correspond to inversions of 5 space coordinates. As we know, each element of the last set produces 16 M-theory 5-branes. Thus we have a total of 48 M-theory 5-branes in the vacuum, each of which appears as a 3-brane in spacetime, as two of its dimensions are wrapped around an internal $T^{2}$.

The actual degrees of freedom carried by these branes will depend on what is projected out from each twisted sector upon requiring invariance under the remaining elements. Before projection, the brane multiplets are gauge multiplets of $N=4$ supersymmetry in $4 \mathrm{~d}$, thus their content is $\left(A_{\mu}, 6 \phi\right)$. Projection will select from this an $N=1$ multiplet, which can be either a vector or a chiral multiplet.

Similarly, the other 4 twisted sectors will each produce a rank-16 gauge bundle, for which the actual gauge group and the multiplets projected out/in have to be determined. Our present knowledge is not yet powerful enough to work this out directly in M-theory and in four dimensions we do not have the constraint of satisfying gravitational anomaly cancellation equations. Going by analogy with the $\mathrm{C}^{\prime}$ model of M-theory on $T^{5} /\left(Z_{2} \times Z_{2}\right)$ and what we have seen in the equivalent description in terms of IIB orientifolds and Ftheory, it seems quite certain that there will be at least two distinct consistent projections for this model in the framework of M-theory.

In fact, we can put forward the following argument in M-theory that these different projections are indeed the manifestation of discrete torsion. In a $\left(Z_{2}\right)^{3}$ orbifold of Mtheory, we could imagine turning on discrete torsion in a $\left(Z_{2}\right)^{2}$ which would be seen in string theory (for instance, the $\left(Z_{2}\right)^{2}$ in the second factor of $S^{1} / Z_{2} \times T^{6} /\left(Z_{2}\right)^{2}$ ). But Lorentz invariance in 11 dimensions can turn the action of these spacetime $Z_{2}$ 's into those with an $\Omega$. This therefore turns what was discrete torsion into a complementary projection in some $\Omega$ twisted sector. A T-duality ensures that this ambiguity exists in the orientifolds of IIB as well. We will see a realisation of this in an example in the next section. It is also clear that in general, all the discrete torsion elements will come into play, indicating a fourfold ambiguity in the definition of our orbifolds/orientifolds. This would come from a twofold ambiguity in $\Omega$ and a twofold conventional discrete torsion.

Finally, for the sake of completeness we list the remaining $T^{8} /\left(Z_{2}\right)^{3}$ orbifolds of Ftheory. Since the generators $\alpha$ and $\beta$ are the same as in the $(C, C)$ case, we only need to list $\gamma$ individually for each of the cases. 
The element $\gamma$ for toroidal fourfolds

\begin{tabular}{|c|c|c|c|c|c|c|c|c|}
\hline & $x^{12}$ & $x^{11}$ & $x^{10}$ & $x^{9}$ & $x^{8}$ & $x^{7}$ & $x^{6}$ & $x^{5}$ \\
\hline$(\mathrm{A}, \mathrm{A})$ &,$- \frac{1}{2}$ & - &,$+ \frac{1}{2}$ & + &,$- \frac{1}{2}$ & - &,$+ \frac{1}{2}$ & + \\
\hline$(\mathrm{B}, \mathrm{B})$ & - & - &,$+ \frac{1}{2}$ & + & - & - &,$+ \frac{1}{2}$ & + \\
\hline$(\mathrm{B}, \mathrm{A})$ & - & - &,$+ \frac{1}{2}$ & + &,$- \frac{1}{2}$ & - &,$+ \frac{1}{2}$ & + \\
\hline$(\mathrm{B}, \mathrm{C})$ & - & - &,$+ \frac{1}{2}$ & + & - & - & + & + \\
\hline$(\mathrm{C}, \mathrm{A})$ & - & - & + & + &,$- \frac{1}{2}$ & - &,$+ \frac{1}{2}$ & + \\
\hline$(\mathrm{C}, \mathrm{B})$ & - & - & + & + & - & - &,$+ \frac{1}{2}$ & + \\
\hline
\end{tabular}

The corresponding M-theory orientifolds are realised as $T^{7} /\left(Z_{2}\right)^{3}$ where again the elements $\alpha$ and $\beta$ are the same as in the $\left(\mathrm{C}^{\prime}, \mathrm{C}^{\prime}\right)$ case defined above, so we only need to list the element $\gamma$.

The element $\gamma$ for $7 \mathrm{~d}$ orientifolds

\begin{tabular}{|c|c|c|c|c|c|c|c|}
\hline & $x^{11}$ & $x^{10}$ & $x^{9}$ & $x^{8}$ & $x^{7}$ & $x^{6}$ & $x^{5}$ \\
\hline$\left(\mathrm{B}^{\prime}, \mathrm{B}^{\prime}\right)$ & - &,$+ \frac{1}{2}$ & - & - & - &,$+ \frac{1}{2}$ & + \\
\hline$\left(\mathrm{B}^{\prime}, \mathrm{C}^{\prime}\right)$ & $+\cdot \frac{1}{2}$ & - & - & - & - & + & + \\
\hline$\left(\mathrm{B}^{\prime}, \mathrm{A}^{\prime}\right)$ & - &,$+ \frac{1}{2}$ & - &,$- \frac{1}{2}$ & - &,$+ \frac{1}{2}$ & + \\
\hline$\left(\mathrm{C}^{\prime}, \mathrm{A}^{\prime}\right)$ & - & + & - &,$- \frac{1}{2}$ & - &,$+ \frac{1}{2}$ & + \\
\hline$\left(\mathrm{C}^{\prime}, \mathrm{B}^{\prime}\right)$ & - & + & - & - & - &,$+ \frac{1}{2}$ & + \\
\hline
\end{tabular}

It should be clear that T-duality transformations in the 9 direction produce mappings between each pair of $\mathrm{F}$ and M-theory compactifications listed above. A T-duality in the 9 and 10 directions takes us to type IIB orientifolds, none of which have been independently invesigated.

\section{Models With Higher Supersymmetry in Four Dimensions}

Although this is somewhat off the main point of this paper, we find it appropriate to discuss F-theory and M-theory compactifications to 4 dimensions with $N>1$ supersymmetry. The first example is a rather simple one: F-theory on $T^{8} / Z_{2}$, where the nontrivial element of $Z_{2}$ acts by reversing all the 8 directions $x^{5}, \ldots, x^{12}$. The orbifold $T^{8} / Z_{2}$ cannot be smoothened to a Calabi-Yau, nevertheless it is a consistent manifold for string compactification. In Ref. [8], it was proposed that IIB on this orbifold is dual to M-theory on $T^{9} / Z_{2}$, while in Ref. 39] it was argued that M-theory on this orbifold has 16 M-theory 
2 -branes in 3 dimensions. This shows that the appropriate analogue of $\chi / 24$, the number of branes required to cancel tadpoles, is 16 in this case.

The same argument thus implies that F-theory on $T^{8} / Z_{2}$ has 163 -branes in 4 dimensions. The resulting theory is $\mathrm{N}=4$ super-Yang-Mills theory, with gauge group $U(1)^{16}$ coming from separated 3-branes, along with further contributions from the untwisted sector. Each 3 -brane carries the complete $\mathrm{N}=4$ gauge multiplet with 6 collective coordinates for its motion along $x^{5}, \ldots, x^{10}$.

By the dualities discussed above, we can relate this to M-theory on $T^{5} / Z_{2} \times T^{2}$. In this picture, the theory has 16 M-theory 5-branes wrapped on $T^{2}$ to form 3 -branes. A further compactification on a circle to 3 dimensions leads to a theory with 165 -branes wrapped on $T^{3}$ to form 2-branes. This is electric-magnetic dual to the compactification of M-theory directly on $T^{8} / Z_{2}$ discussed in [39], where one has 16 fundamental M-theory 2-branes rather than wrapped 5-branes. Thus the resulting picture is perfectly consistent.

A more interesting example is F-theory on $T^{8} /\left(Z_{2} \times Z_{2}\right)$ where the orbifolding group is defined to be generated by the elements $\alpha$ and $\beta$ that we used in defining the (C,C) model in the previous section and thus actually is $T^{4} / Z_{2} \times T^{4} / Z_{2}$. This becomes the orientifold of type IIB on $T^{6} /\left\{1, I_{5678}, \Omega(-1)^{F_{L}} I_{910}, \Omega(-1)^{F_{L}} I_{5678910}\right\}$. A T-duality in the 9 direction maps this to type IIA on $T^{6} /\left\{1, I_{5678}, \Omega I_{10}, \Omega I_{567810}\right\}$. Interchanging the 10 and 11 directions gives M-theory on $T^{7} /\left(Z_{2} \times Z_{2}\right)$ where now the group is defined through $\alpha$ and $\beta$ of the $\left(\mathrm{C}^{\prime}, \mathrm{C}^{\prime}\right)$ model. Clearly this is just M-theory compactified on model $\mathrm{C}^{\prime}$ to 6 dimensions, and then further on $T^{2}$ to 4 dimensions. By another pair of Tdualities, this is also equivalent to type IIA on the orbifold limit of the $(51,3)$ Calabi-Yau 3-fold, to 4 dimensions, with $\mathrm{N}=2$ supersymmetry. This last is precisely the case where discrete torsion in string theory was studied 35] [36 We will see that this ambiguity can be mapped onto an orientifolding ambiguity. For that we note that a T-duality $\left(T_{56}\right)$ takes the original IIB orientifold into IIB on $T^{6} /\left\{1, I_{5678}, \Omega I_{56910}, \Omega I_{78910}\right\}$.

Instead let us start out with the particular $T^{7} /\left(Z_{2}\right)^{2}$ orientifold of M-theory, which, in IIA language, would be IIA on $T^{6} /\left\{1,(-1)^{F_{L}} I_{78910}, I_{5678},(-1)^{F_{L}} I_{56910}\right\}$. We immediately see that $T_{10}$ gives us IIB on the $(51,3)$ Calabi-Yau. In the absence of discrete torsion, this is distinct from IIA on this orbifold, which we just encountered. However, turning on discrete torsion in the IIA theory makes it the same as the IIB theory (or vice versa). We will now map this M-theory/IIA orientifold into what is apparently the same IIB orientifold in the last line of the previous paragraph. To do so, we use the Lorentz 
invariance of M-theory to interchange directions 5 and 11. This obtains for us the orientifold, IIA on $T^{6} /\left\{1, \Omega I_{578910}, \Omega(-1)^{F_{L}} I_{678},(-1)^{F_{L}} I_{56910}\right\}$. Finally, $T_{5}$ results in IIB on $T^{6} /\left\{1, \Omega I_{78910}, \Omega I_{5678}, I_{56910}\right\}$, which is the claimed orientifold (after a relabelling of $7,8$ as 9,10$)$. Thus we see that the ambiguity of discrete torsion in string theory on the $(51,3)$ orbifold has manifested itself in an ambiguity in the (equivalent) orientifold of IIB on $T^{6} /\left\{1, I_{5678}, \Omega I_{56910}, \Omega I_{78910}\right\}$. The two different projections give us either IIA or IIB on the $(51,3)$.

One can similarly generate $\mathrm{N}=2$ compactifications of $\mathrm{M}$ and F-theory to 4 dimensions which correspond to type IIA on the $(11,11)$ or $(19,19)$ Calabi-Yau 3-folds, by picking a suitable $Z_{2} \times Z_{2}$ subgroup of the $\left(Z_{2}\right)^{3}$ group of the previous section for the $(\mathrm{C}, \mathrm{A})$ and $(\mathrm{C}, \mathrm{B})$ models.

Although this discussion concerns compactifications with higher supersymmetry, it is worth noting here for completeness that there is an orbifold with lower supersymmetry than $\mathrm{N}=1$, obtained by starting with model $(\mathrm{C}, \mathrm{C})$ of the previous section and adjoining the generator $\delta$ with acts as $(+,-,+,-,+,-,+,-)$. This is precisely the $T^{8} /\left(Z_{2}\right)^{4}$ orbifold which leads to the Joyce 8 -manifold of $\operatorname{spin}(7)$ holonomy 40]. The extra generator kills another half of the supersymmetry, leading apparently to an $\mathrm{N}=\frac{1}{2}$ compactification of $\mathrm{F}$ theory. Unfortunately, as is evident from the structure of $\delta$, the action on the fibre (the first two coordinates) is not in $\mathrm{SL}(2, \mathrm{Z})$, so this is not a valid compactification of F-theory at all, avoiding a potential paradox. It is, however, a valid orbifold for M-theory to three dimensions, where it leads to $\mathrm{N}=1$ supersymmetry [13].

\section{Discussion and Conclusions}

We have defined a class of orbifolds of F-theory and, in one-to-one correspondence, a class of orientifolds of M-theory, which lead to $\mathrm{N}=1$ supersymmetry in 6 and 4 spacetime dimensions. The totality of available examples in the latter class is of the order of a few hundred. From the F-theory point of view, the examples are all of the form $\left(K 3 \times T^{2}\right) / Z_{2}$ (to six dimensions) and $(K 3 \times K 3) / Z_{2}$ (to four dimensions).

We have been able to identify the spectra of these models in a number of cases, and shown that they pass various consistency checks. One relatively surprising discovery is the presence of an ambiguity, analogous to discrete torsion, in those models where

1 The contents of this paragraph were essentially explained to us by E. Witten. 
the orbifolding group has a sufficient number of elements with fixed points. In the sixdimensional case, we related this to the possibility of defining two distinct actions of the orientation-reversal operator $\Omega$ in orientifold models. In four dimensions we predict a larger ambiguity, coming from a combination of conventional discrete torsion and the ambiguity discussed above.

This may eventually lead to the discovery of some new property inherent in $\mathrm{M}$ and $\mathrm{F}$ theories, for the following reason. F-theory, in particular, can be interpreted (though not yet with complete success) as a 12-dimensional theory compactified on a Calabi-Yau p-fold. In the simplest case where the ambiguity appears, it relates F-theory on two Calabi-Yau 3-folds, with Hodge numbers $(51,3)$ and $(3,243)$, which do not appear to be related by a corresponding ambiguity in string theory. The known ambiguity in string theory relevant to the present case relates instead the $(51,3)$ to the $(3,51)$, and has been interpreted at least in this special case as mirror symmetry. In contrast, F-theory cannot be compactified on the $(3,51)$ since the result does not satisfy $6 \mathrm{~d}$ anomaly cancellation. We might try to generalise from the behaviour of 4 dimensional string theories with discrete torsion at conifold singularities. The resulting theory is non-singular. The theory without discrete torsion would however have been singular and we now understand that there are massless particles and the theory undergoes a phase transition. The analogous phase transitions in six dimensions are mediated by tensionless strings. Indeed, the $(51,3)$ can undergo an extremal transition to the $(3,243)$ from the shrinking of 16 2-cycles in the base. These give rise to tensionless strings. Perhaps the presence of the analogue of discrete torsion prevents the theory from making this transition. This clearly needs to be studied further.

From the point of view of both M and F-theory, it is evident that once the orbifold group is larger than $Z_{2}$, one has to find a precise way to determine how twisted-sector states are projected in or out by the other elements. Besides the bearing of this question on the ambiguity discussed above, it is also of interest in that it may help uncover the basic structure underlying these theories, beyond the important discovery [9] [2] that twisted-sector states in these theories frequently arise from condensation of branes. This discrete-torsion-like behaviour in M-theory, is another indication, like the appearance of twisted sectors, that M-theory orbifolds show some similar behaviour to that of conformal field theories.

A very important point that emerged recently from the work of Sen[16] is that although F-theory in an orbifold limit is dual to a type IIB orientifold, the naive moduli space that would be suggested by perturbative study of the orientifold is quite different from the exact 
moduli space, which is successfully predicted by F-theory in the case studied. It seems likely that the nontrivial relation between the two persists in the cases we have considered here, namely compactifications to 6 and 4 dimensions. (This ultimately suggests that Ftheory is the most powerful of the various formulations, though ironically it is also the one whose foundations are the least well-understood. This is also supported by the fact that all M-theory orbifolds (at least of the $Z_{2}$ variety) seem to have a realisation in F-theory.)

Given the relative solvability of orbifold compactifications in string theory, one may treat the Borcea 4-fold examples that we have presented as laboratories to study a wide variety of physical effects: phase transitions [42] and nonperturbative superpotentials [43] in particular. We hope to report on such investigations in the future. The study of F-theory compactifications to 4 dimensions (which has been initiated in the above references and in Refs. 25, 44 is still in its infancy, and it is likely that it will lead to a new understanding of physically interesting string compactifications.

Note Added: After the work presented in Sections 2 and 3 was completed, some preprints appeared which overlap with a few of the observations contained therein. In particular, Ref. 45] talks about realizations of some $\mathrm{N}=16 \mathrm{~d}$ models in terms of M-theory, F-theory and string theory. Two more papers, Refs. 46] [47, confirm that there exists a consistent variant of the Gimon-Polchinski model for which $\Omega^{2}=1$, whose spectrum corresponds with that of F-theory on the $(51,3)$ Calabi-Yau. (In the GP model, one has $\Omega^{2}=-1$ on certain twisted-sector states.)

Acknowledgements We are grateful to J. Blum, A. Dabholkar, O.Ganor, D.Gross, P. Horava, D. Morrison, J. Polchinski, J. Schwarz, C. Vafa, A. Zaffaroni and especially A. Sen and E. Witten for helpful discussions. One of us (S.M.) acknowledges the hospitality of the Institute for Advanced Study, Princeton, and the Physics Department at Caltech, where part of this work was done. 


\section{References}

[1] E. Witten, hep-th/9503124, Nucl. Phys. B443 (1995) 85.

[2] C. Vafa, "Evidence for F-Theory" hep-th/9602022.

[3] L. Dixon, J. Harvey, C. Vafa and E. Witten, Nucl. Phys. B261 (1985) 678, Nucl. Phys. B274 (1986) 285.

[4] P. Horava, Nucl. Phys. B327 (1989) 461.

[5] A. Sagnotti, "Open Strings and Their Symmetry Groups", in Non-perturbative Quantum Field Theory, Cargese 1987, eds. G. Mack et. al. (Pergamon Press 1988);

P. Horava, Phys. Lett. B231 (1989) 251;

J. Dai, R.G. Leigh and J. Polchinski, Mod. Phys. Lett. A4 (1989) 2073.

[6] P. Horava and E. Witten, hep-th/9510209, Nucl. Phys. B460 (1996) 506.

[7] A. Sen, hep-th/9603113, Mod. Phys. Lett. A11(1996) 1339.

[8] K. Dasgupta and S. Mukhi, hep-th/9512196, Nucl. Phys. B465 (1996) 399.

[9] E. Witten, hep-th/9512219, Nucl. Phys. B463 (1996) 383.

[10] A. Sen, hep-th/9602010, Phys. Rev. D53 (1996) 6725.

[11] A. Kumar and K. Ray, hep-th/9602144, Phys. Rev. D, to appear.

[12] A. Kumar, K. Ray, "Compactification of M-Theory to Two Dimensions", hepth/9604164.

[13] B.S. Acharya, "N=1 M-Theory Heterotic Duality in Three-Dimensions and Joyce Manifolds", hep-th/9604133.

[14] B.S. Acharya, "M-Theory Compactification and Two Brane/Five Brane Duality", hepth/9605047.

[15] D. Morrison and C. Vafa, "Compactifications of F-Theory on Calabi-Yau Threefolds (II)", hep-th/9603161.

[16] A. Sen, "F-Theory and Orientifolds", hep-th/9605150.

[17] K. Dasgupta and S. Mukhi, "F-Theory at Constant Coupling", hep-th/9606044.

[18] E. Gimon and J. Polchinski, hep-th/9601038, Phys. Rev. D, to appear.

[19] A. Dabholkar and J. Park, "An Orientifold of Type IIB Theory on K3", hepth/9602030.

[20] E. Gimon and C. Johnson, "K3 Orientifolds", hep-th/9604129.

[21] A. Dabholkar and J. Park, "Strings on Orientifolds", hep-th/9604178.

[22] M. Berkooz and R. Leigh, " $A D=4$ N=1 Orbifold of Type I Strings", hep-th/9605049.

[23] M. Berkooz, R. Leigh, J. Polchinski, J.H. Schwarz, N. Seiberg and E. Witten, "Anomalies, Dualities, and Topology of $D=6 N=1$ Superstring Vacua", hep-th/9605184.

[24] J. Polchinski, "Tensors From K3 Orientifolds", hep-th/9606165.

[25] S. Sethi, C. Vafa and E. Witten, "Constraints on Low-Dimensional String Compactifications", hep-th/9606122. 
[26] C. Voisin, in Journées de Géometrie Algébrique d'Orsay, Astérisque No. 218 (1993) 273.

[27] C. Borcea, "K3 Surfaces with Involution and Mirror Pairs of Calabi-Yau Manifolds", to appear in Essays on Mirror Manifolds II.

[28] M. Bershadsky, K. Intriligator, S. Kachru, D. Morrison, V. Sadov and C. Vafa, "Geometric Singularities and Enhanced Gauge Symmetries", hep-th/9605200.

[29] S. Ferrara, J. Harvey, A. Strominger and C. Vafa, hep-th/9505162, Phys. Lett. B361 (1995) 59.

[30] M. Duff, R. Minasian and E. Witten, "Evidence For Heterotic/Heterotic Duality", hep-th/9601036.

[31] D. Morrison and C. Vafa, "Compactifications of F-Theory on Calabi-Yau Threefolds $1 "$, hep-th/9602114.

[32] J.H. Schwarz and A. Sen, hep-th/9507027, Phys. Lett. B357 (1995) 323.

[33] S. Chaudhuri and D. Lowe, hep-th/9508144, Nucl. Phys. B459 (1995) 113.

[34] C. Vafa, Nucl. Phys. B273 (1986) 592.

[35] A. Font, L.E. Ibanez and F. Quevedo, Phys. Lett. B217 (1989) 272.

[36] C. Vafa and E. Witten, hep-th/9409188, J. Geom. Phys. 15 (1995) 189.

[37] P. Aspinwall and D. Morrison (appendix by M. Gross), hep-th/9503208, Comm. Math. Phys. 178 (1996) 115.

[38] V. Nikulin, in Proceedings of the International Congress of Mathematicians, Berkeley, (1986) 654.

[39] A. Sen, "Duality and Orbifolds", hep-th/9604070.

[40] D. Joyce, "Compact Riemannian Manifolds with Holonomy Spin(7)", to appear in Inv. Math.

[41] N. Seiberg and E. Witten, "Comments on String Dynamics in Six-Dimensions", hepth/9603003.

[42] E. Witten, "Phase Transitions in M-Theory and F-Theory", hep-th/9603150.

[43] E. Witten, "Nonperturbative Superpotentials in String Theory", hep-th/9604030.

[44] I. Brunner and R. Schimmrigk, "F-Theory on Calabi-Yau Fourfolds", hep-th/9606148.

[45] E. Gimon and C. Johnson, "Multiple Realizations of N=1 Vacua in Six-Dimensions", hep-th/9606176.

[46] J. Blum and A. Zaffaroni, "An Orientifold from F-Theory", hep-th/9607019.

[47] A. Dabholkar and J. Park, "A Note on Orientifolds and F-Theory", hep-th/9607041. 